\title{
The Solution to the Problem of Time in Shape Dynamics
}

\author{
Julian Barbour, ${ }^{1,2}$ Tim Koslowski ${ }^{3}$ and Flavio Mercati ${ }^{4}$ \\ ${ }^{1}$ College Farm, South Newington, Banbury, Oxon, OX15 4JG UK, \\ ${ }^{2}$ Visiting Professor in Physics at the University of Oxford, UK \\ ${ }^{3}$ University of New Brunswick, Fredericton, NB, E3B 5 A3 Canada, \\ ${ }^{4}$ Perimeter Institute for Theoretical Physics, 31 Caroline Street North, \\ Waterloo, ON, N2L $2 Y 5$ Canada.
}

\begin{abstract}
The absence of unique time evolution in Einstein's spacetime description of gravity leads to the hitherto unresolved 'problem of time' in quantum gravity. Shape Dynamics is an objectively equivalent representation of gravity that trades spacetime refoliation invariance for three-dimensional conformal invariance. Its logical completion presented here gives a dimensionless description of gravitational dynamics. We show that in this framework the classical problem of time is completely solved. Since a comparable definitive solution is impossible within the spacetime description, we believe Shape Dynamics provides a key ingredient for the creation of quantum gravity.
\end{abstract}

\section{Introduction}

In the canonical formulation of General Relativity due to Arnowitt, Deser and Misner $(\mathrm{ADM})$, the total Hamiltonian is a pure constraint:

$$
\mathcal{H}=\int \mathrm{d} t \int \mathrm{d}^{3} x\left(N(x, t) H(x)-2 N_{i}(x, t) \nabla_{j} p^{i j}\right),
$$

in the case of spatial slices that are compact without boundary. Variation wrt the position-dependent lapse $N$ gives at each space point the constraint

$$
H=\frac{1}{\sqrt{g}}\left(p^{i j} p_{i j}-\frac{1}{2} p^{2}\right)-\sqrt{g} R=0,
$$

where $p^{i j}$ are the momenta conjugate to the 3 -metric $g_{i j}, p=g_{i j} p^{i j}$, and $g=\operatorname{det} g_{i j}$. Variation wrt the shift $N_{i}$ leads to the linear ADM momentum constraint

$$
-2 \nabla_{j} p^{i j}=0
$$

It generates 3-diffeomorphisms and is a proper gauge constraint. In contrast, the quadratic constraint (2) evolves the system but at arbitrarily different rates at each space point depending on the freely specifiable lapse $N$ (many-fingered time). This 
lack of determinate evolution arises from the refoliation invariance of General Relativity (GR) in the spacetime representation and for quantization creates the severe and long unresolved problem of time [1]. It has two core aspects.

1. Unlike (3), the Hamiltonian constraints (2) do not arise from the action of a group on configuration space, which is why GR fails to satisfy the Mach-Poincaré principle (see below) in either its strong or weak form. Instead, as Dirac noted long ago [2], the Hamiltonian constraints entangle gauge invariance with true dynamics. Shape Dynamics (SD) disentangles them, without introducing either second class constraints or additional degrees of freedom, by replacing the Hamiltonian constraints with local three-dimensional conformal constraints and a single volume constraint that acts as the generator of dynamics. This eliminates many-fingered evolution.

2. Even in a particular foliation, a quantum problem remains. The theory is still reparametrization invariant with a residual global constraint corresponding to a vanishing Hamiltonian. This feature is not specific to GR and arises in any theory with a quadratic constraint. Traditional quantization automatically leads to a timeless model with a time-independent Schrödinger equation. This is the quantum 'frozen-formalism' problem of time. We solve it by finding a dimensionless and scale-invariant system with a preferred independent variable, ${ }^{1} \tau$, that makes predictions indistinguishable from the objective predictions of GR. The transition to a representation in which both the true dynamical degrees of freedom and, in our key innovation, $\tau$ are dimensionless is the logical completion of SD and simultaneously the solution to the problem of time.

\section{Approaches to the Problem of Time}

To highlight the new elements we bring to the issue, we briefly review the most sophisticated current line of attack, the 'effective approach' [3]. A timeless quantum universe is accepted as fundamental and assumed to be in a semiclassical highquantum-numbers regime with its wavefunction $\Psi$ peaked around a classical trajectory. One physical observable is singled out as an internal time wrt which $\Psi$ evolves. Since no observable possesses everywhere the 'good-clock' requirement of monotonicity, a 'grasshopper' strategy is adopted: the internal time 'jumps' from one variable to another on the approach to a turning point.

While the universe may well get into a semiclassical regime, peaking of $\Psi$ around a particular classical trajectory is a much stronger assumption. Will the required 'unquantizing' of a degree of freedom occur?

A much simpler solution would be one in which, just as in existing quantum mechanics, time is sui generis, i.e., quite different from the quantum degrees of freedom because it is a genuine independent variable. We shall show that such a variable does exist in a spatially closed universe and moreover is monotonic.

The demonstration relies on two facts. First, the objectively equivalent shapedynamic representation of Einstein's theory shows that there is a mathematically and physically well-defined notion of simultaneity within closed-space GR. This

\footnotetext{
${ }^{1}$ We say 'independent variable' rather than 'time' because, as explained in footnote $8, \tau$ has no relation at all to the readings of physical clocks.
} 
solves the foliation problem. Second, the shape-dynamic variables divide unambiguously into two kinds: dimensionless true degrees of freedom and the single monotonic dimensionless independent variable $\tau$.

\section{Shape Dynamics}

Shape Dynamics has been developed in stages over more than 30 years $[4,5,6,7]$. It is a general framework for all closed dynamical systems in which there exists a reduced configuration space S (Shape Space) of physical degrees of freedom that, together with the independent variable, are all dimensionless. This is the logical completion referred to in the abstract.

Its foundation is the Mach-Poincaré principle [8, 4, 9]: Initial data in $\mathbf{S}$ in the form of a point and a direction (strong form) or a point and tangent vector (weak form) must determine the physical evolution curve in $S$ uniquely.

General Relativity fails to realize the Mach-Poincaré principle in Wheeler's superspace $W=$ Riem/Diff [10,11]: given a point and direction (or tangent vector) in W there is a whole sheaf of solutions to the ADM equations of motion, each corresponding to a different foliation of one and the same spacetime. York's work on the initial-value problem in GR $[12,13]$ hinted that conformal superspace $\mathrm{C}$, the space of conformal 3-geometries, is the physical configuration space of gravity, not W. This does not quite work: the implicit requirement of full conformal invariance freezes the dynamics. One is forced to a less strict notion of what is dynamical and to allow the total volume of a compact 3-geometry, $V=\int d^{3} x \sqrt{g}$, to evolve $[6,14,7]$. Then the strong Mach-Poincaré principle is satisfied on conformal superspace plus volume, with a single simultaneity-defining global Hamiltonian constraint. The final step to the logical completion of SD, anticipated in [15] and carried out explicitly in this paper, is to deparametrize the residual global constraint. This completes the solution to the problem of time.

We expect that many readers, convinced by Einstein and Minkowski that universal simultaneity cannot be meaningfully defined, will balk at our solution. However, we emhasize that, despite its ontologically preferred time, SD creates the familiar spacetime picture on-shell. It therefore reproduces all the confirmed predictions of GR and moreover gives a much simpler account of gravitational dynamics: one shape of the universe succeeds another along a unique evolution curve in S.

\section{The $N$-Body Quantum Problem of Time}

We use the Barbour-Bertotti (BB) formulation of the $N$-body problem $[4,16]$ as a toy model to illustrate our key innovation, the insistence that both the independent variable and the observables be dimensionless. The BB extended configuration space is $\mathbb{R}^{3 N} ; \mathbf{r}_{a}=\left(r_{a}^{1}, r_{a}^{2}, r_{a}^{3}\right) \in \mathbb{R}^{3}$ are the Cartesian coordinates of particle $a \in\{1, \ldots, N\}$. Newton's absolute space is eliminated by a reduction to $\mathrm{Q}^{N}=$ $\mathbb{R}^{3 N} /$ Eucl, where Eucl is the Euclidean group of rigid translations and rotations [4]. Newton's absolute time is replaced by a path label $\lambda$ in a geodesic principle on $\mathrm{Q}^{N}$. The resulting theory is invariant under $\lambda$-dependent Eucl transformations $[4,16]$ and 
reproduces Newtonian gravity but with the further predictions that

$$
\mathbf{P}=\sum_{a=1}^{N} \mathbf{p}^{a}=0, \quad \mathbf{L}=\sum_{a=1}^{N} \mathbf{r}_{a} \times \mathbf{p}^{a}=0
$$

where $\mathbf{p}^{a}$ are canonical momenta $\left\{r_{a}^{i}, p_{j}^{b}\right\}=\delta_{a}{ }^{b} \delta^{i}{ }_{j}$, and $\mathbf{P}$ and $\mathbf{L}$ are the total linear and angular momenta of the system. The linear constraints (4) taken together are analogous to the ADM momentum constraint (3). The geodesic principle on $\mathrm{Q}^{N}$ gives rise to a quadratic reparametrization constraint that fixes the magnitude of the canonical momenta $\mathbf{p}^{a}$ and is the counterpart of ADM's (2):

$$
H=\sum_{a=1}^{N} \frac{\mathbf{p}^{a} \cdot \mathbf{p}^{a}}{2 \mu_{a}}-E+V_{\text {New }}=0 .
$$

Here $\mu_{a}=m_{a} / M, M=\sum_{b} m_{b}$, are dimensionless 'geometrical' masses, and $V_{\text {New }}$ is the Newton potential:

$$
V_{\mathrm{New}}=-\sum_{a<b} \frac{\mu_{a} \mu_{b}}{r_{a b}}, \quad r_{a b}=\left\|\mathbf{r}_{b}-\mathbf{r}_{a}\right\|
$$

Note that $\left[V_{\text {New }}\right]=\ell^{-1}$ (there are no time or mass dimensions in our dynamical variables), $\left[\mathbf{r}_{a}\right]=\ell$ and (from (5)) $\left[\mathbf{p}^{a}\right]=\ell^{-\frac{1}{2}}$.

To implement the Mach-Poincaré principle, we postulate that only invariants of the similarity group Sim (Eucl plus dilatations) are true Lagrangian degrees of freedom (from which, we anticipate, quantum Dirac observables are to be constructed) and reduce our configuration space to Shape Space $S=\mathbb{R}^{3 N} / \operatorname{Sim}$. The scale-invariance constraint analogous to (4) would be vanishing of the dilatational momentum $D$

$$
D=\sum_{a=1}^{N} \mathbf{r}_{a} \cdot \mathbf{p}^{a}=0 .
$$

We say 'would be' because, unlike the constraints (4), $D=0$ is not conserved by the Newton potential (6), which is invariant under translations and rotations but not dilatations. In fact, $2 D \approx \dot{I}_{\mathrm{cm}}$ is the time derivative of the center-of-mass moment of inertia $I_{\mathrm{cm}}$,

$$
I_{\mathrm{cm}}=\sum_{a=1}^{N} \mu_{a}\left\|\mathbf{r}_{a}^{\mathrm{cm}}\right\|^{2} \equiv \sum_{a<b} \mu_{a} \mu_{b} r_{a b}^{2},
$$

where $\mathbf{r}_{a}^{\mathrm{cm}}=\mathbf{r}_{a}-\sum_{b} \mu_{b} \mathbf{r}_{b}$. Note that $R=I_{\mathrm{cm}}^{1 / 2}$ measures the root-mean-square length, or scale, of the system $\left(\left[I_{\mathrm{cm}}^{1 / 2}\right]=\ell\right)$. Thus, whereas $\mathbf{P}$ and $\mathbf{L}$, which measure unobservable overall change of position and orientation in Newton's absolute space, can be made to vanish in $\mathrm{BB}$, this cannot be done for the overall expansion rate $D$.

But total scale is purely conventional in a closed universe. Expansion of the universe is not seen but deduced - from comparison of simultaneously observed wavelengths. One way to solve this problem is to change the potential in such a way so as to ensure that the constraint (7) is conserved. The resulting theory then satisfies the strong Mach-Poincaré principle and, as shown in [17], has interesting 
quantum consequences, in particular, a breakdown of scale invariance due to a quantum anomaly. Here we take a different route, presenting a theory which satisfies only the weak Mach-Poincaré principle but is still scale invariant.

At any instant the Newtonian centre-of-mass kinetic energy $T$ decomposes uniquely into three terms [18]: $T=T_{\mathrm{r}}+T_{\mathrm{d}}+T_{\mathrm{s}}$, the rotational, dilatational and shape parts, respectively. We seek a theory with only $T_{\mathrm{s}}$. The constraints (4) eliminate $T_{\mathrm{r}}$ and the translational part $T_{\mathrm{p}}$. In the next step, instead of changing the potential, we retain $E-V_{\text {New }}$ but swap the reparametrization invariance of (5) for a time $\tau$ that subsumes $T_{\mathrm{d}}$.

Our $\tau$ will be monotonic thanks to an important fact: if in any dynamical system the potential $V$ is homogeneous of some degree $k$, Euler's homogeneous-function theorem and Newton's second law imply $\ddot{I}_{\mathrm{cm}}=4(E-V)-2 k V$. Since $k=-1$ for $V_{\mathrm{New}}$, this becomes $\ddot{I}_{\mathrm{cm}}=4 E-2 V_{\mathrm{New}}$. Now $V_{\mathrm{New}}$ is negative, so if, as we shall assume, $E \geq 0$, then the function $I_{\mathrm{cm}}(t)$ is concave upward and $D$ monotonic. This is not generically true of any physical observable. We already noted that scale, like time, is not observable; we now see that, through the monotonicity of $D$, scale shares another defining property of Newton's time $t$.

Homogeneity of the potential has another important consequence: dynamical similarity [19]. Let a solution of the dynamical equations be given and the coordinates and time be scaled with a constant $C$ as follows: $\mathbf{r}_{a} \rightarrow C \mathbf{r}_{a}, t \rightarrow C^{1-k / 2}$. Then geometrically similar paths are obtained. The elapsed times at which corresponding points are reached are in the ratio $t^{\prime} / t=\left(l^{\prime} / l\right)^{1-k / 2}$. For the Newton potential with $k=-1$, this yields Kepler's third law.

Using the example of Newtonian dynamics, we can illustrate the way in which a given physical theory can be represented in two very different ways. Let solutions of Newton's equations be generated in $\mathbb{R}^{3 N}$ and then 'projected' to Shape Space by abstraction of everything defined by conventional choice of units and the origin and orientation of the coordinate axes. Then the Newtonian solutions $\mathbf{r}_{a}(t)$ are mapped to a curve of shapes $s(\lambda)$ in $\mathrm{S}$. We call this the passage from the coordinatized to the objective representation. The latter eliminates all redundancy and human convention from the description of the dynamics. The description is dimensionless and retains only what is objective: as we have seen, when the potential is homogeneous, a seemingly distinct one-parameter family of solutions in the coordinatized repesentation is mapped to a single objective curve in S.

We now show how a suitably defined $\tau$ eliminates the dilatational part $T_{\mathrm{d}}$. We should derive equations in $\mathrm{S}$, but, the non-Abelian rotations being difficult, we quotient only wrt dilatations and translations, which takes us to 'pre-shape space' $\mathrm{PS}=\mathbb{R}^{3 N-3} /$ Dil $\times$ Transl, on which we introduce the 'gauge-fixed' coordinates and momenta

$$
\boldsymbol{\sigma}_{a}=\sqrt{\mu_{a}} \frac{\mathbf{r}_{a}^{\mathrm{cm}}}{R}, \quad \boldsymbol{\pi}^{a}=\frac{1}{\sqrt{\mu}_{a}} \frac{R}{D_{0}} \mathbf{p}_{\mathrm{cm}}^{a}-\sqrt{\mu_{a}} \frac{D}{D_{0}} \boldsymbol{\sigma}_{a}, \quad R=I_{\mathrm{cm}}^{1 / 2} .
$$

Here $\boldsymbol{\sigma}_{a}$ coordinatize a unit $(3 N-4)$-dimensional sphere, and $\boldsymbol{\pi}^{a}$ are the $3 N-4$ momenta tangent to that sphere divided by the value $D_{0}$ of $D$ at the point chosen to begin evolution. ${ }^{2}$ This division by $D_{0}$ makes the momenta dimensionless like $\boldsymbol{\sigma}_{a}$.

\footnotetext{
${ }^{2}$ In the many equivalent coordinatized representations, $D_{0}$ will have different values, but since
} 
We have

$$
\begin{aligned}
& \sum_{\mathrm{a}=1}^{\mathrm{N}} \boldsymbol{\sigma}_{a} \cdot \boldsymbol{\sigma}_{a}=1, \quad \sum_{\mathrm{a}=1}^{\mathrm{N}} \boldsymbol{\pi}^{a} \cdot \boldsymbol{\sigma}_{a}=0, \\
& \sum_{\mathrm{a}=1}^{\mathrm{N}} \sqrt{\mu_{a}} \boldsymbol{\sigma}_{a}=0, \quad \sum_{\mathrm{a}=1}^{\mathrm{N}} \sqrt{\mu_{a}} \boldsymbol{\pi}^{a}=0 .
\end{aligned}
$$

The constraints (10) are first class among themselves wrt the Hamiltonian constraint (5).

We now express the Newton potential as $V_{\text {New }}(\mathbf{r})=V_{\mathrm{s}}(\boldsymbol{\sigma}) / R$, calling $V_{\mathrm{s}}$ the shape potential; it is a function on Shape Space. ${ }^{3}$ The kinetic energy decomposes into the dilatational part, $T_{\mathrm{d}}=\frac{1}{2} D^{2} / R^{2}$, and shape part $T_{\mathrm{s}}=\frac{1}{2} D_{0}^{2} K_{\mathrm{s}} / R^{2}$, where $K_{\mathrm{s}}=\sum_{a=1}^{N} \boldsymbol{\pi}^{a} \cdot \boldsymbol{\pi}^{a}$. The $\left(\boldsymbol{\sigma}_{a}, \boldsymbol{\pi}^{b}\right)$ coordinates are scale invariant, as they Poisson commute with $D$ and $R$,

$$
\left\{f(D, R), \boldsymbol{\pi}^{a}\right\}=\left\{f(D, R), \boldsymbol{\sigma}_{a}\right\}=0,
$$

These relations show that the associated Dirac bracket is block diagonal, which is important for the deparametrization performed below.

The symplectic structure on pre-shape space is

$$
\begin{aligned}
& \left\{\sigma_{i}^{a}, \pi_{b}^{j}\right\}=D_{0}^{-1}\left(\delta^{a}{ }_{b} \delta^{j}{ }_{i}-\sigma_{a}^{i} \sigma_{b}^{j}\right), \\
& \left\{\pi_{i}^{a}, \pi_{j}^{b}\right\}=D_{0}^{-1}\left(\sigma_{a}^{i} \pi_{j}^{b}-\sigma_{b}^{j} \pi_{i}^{a}\right), \\
& \left\{\sigma_{i}^{a}, \sigma_{b}^{j}\right\}=0 .
\end{aligned}
$$

We now introduce the monotonic and dimensionless independent variable $\tau=$ $D / D_{0}$ and obtain an unconstrained (true) Hamiltonian. The $\log$ of $R$ is canonically conjugate to $D,\{D, \log R\}=1$. Therefore $\mathcal{H}=-\log \left(R / R_{0}\right)+$ const can be used as the generator of $\tau$-translations: take a shape observable $f=f(\boldsymbol{\sigma}, \boldsymbol{\pi})$, its evolution wrt $D$ is

$$
\frac{\partial f}{\partial \tau}=D_{0}\{\mathcal{H}, f\}
$$

Note that the $D_{0}$ on the rhs will cancel the $D_{0}^{-1}$ in (12), removing from the equations of motion all dependence on $D_{0}$, the only dimensionful quantity remaining in the theory. As is required for the objective description of a closed universe, the equations of motion are dimensionless.

The innovation of introducing a dimensionless time is, in our view, essential for the satisfactory passage from a constrained to a physical Hamiltonian. We are not aware that it has been considered hitherto. It leads to a notion of time very different from that of either Newton or Einstein. The origin of $\tau$ is conventional - it can be taken at any point along the orbit in Shape Space. After that $\tau$ changes at a definite rate along the orbit from the origin as $R / R_{0}$ changes. ${ }^{4}$ Besides monotonicity, the advantage of using $D$ to define $\tau$ is its status as a uniquely distinguished collective variable associated with the overall behaviour of the system. The growth of $\tau$ is

we always take dimensionless ratios the same quantities are invariably obtained in the objective description. It is important that the point in $\mathbb{R}^{3 N}$ at which the evolution is begun, where $D=D_{0}$, corresponds to one and the same shape $s_{0} \in \mathrm{S}$.

${ }^{3}$ As pointed out in [17], the dimensionless $V_{\mathrm{s}}$ is not only the potential that governs the scaleinvariant dynamics but also an objective measure of gravitational complexity; we anticipate that it will be the most important Dirac observable in the quantum treatment.

${ }^{4}$ We should point out that any definite monotonic function of $D / D_{0}$ can also be chosen as time. We shall return to this point in footnote 8 . 
created by all the physical degrees of freedom working together. This enables us to avoid arbitrary choice of individual physical degrees of freedom as 'time' with the inevitable 'hopping' from one to another.

From the conceptual point of view, it is also important that, interpreted in the usual scale-dependent coordinatized description, our variable $\tau$ appears as an internal time, but it is an external evolution parameter in the scale-invariant dimensionless description. Since it is the latter which represents the objective state of affairs, the distinction, which appears in exactly the same way in geometrodynamic SD and will be a central feature in the quantum treatment, needs to be made.

To find the physical Hamiltonian $\mathcal{H}$ we solve the quadratic constraint (5) for $R$ :

$$
R^{2} E-R V_{\mathrm{s}}-\frac{1}{2} D^{2}-\frac{1}{2} D_{0}^{2} K_{\mathrm{s}}=0 .
$$

In the simplest case when $E=0$ the Hamiltonian is

$$
\mathcal{H}=\log \frac{K_{\mathrm{s}}+\tau^{2}}{\left|V_{\mathrm{s}}\right|},
$$

and the equations of motion it generates are (notice the disappearance of $D_{0}$ ):

$$
\frac{d \boldsymbol{\sigma}_{a}}{d \tau} \approx \frac{2 \boldsymbol{\pi}^{a}}{K_{\mathrm{s}}+\tau^{2}}, \frac{d \boldsymbol{\pi}^{a}}{d \tau} \approx \frac{\partial \log \left|V_{\mathrm{s}}\right|}{\partial \boldsymbol{\sigma}_{a}}-\frac{K_{\mathrm{s}}}{K_{\mathrm{s}}+\tau^{2}} \boldsymbol{\sigma}_{a} .
$$

Quotienting wrt rotations would not change anything essential, as all our equations are rotationally invariant.

The system of equations on Shape Space that we have obtained, (16), is not autonomous. But this is not in any way analogous to explicit time dependence in laboratory physics induced by, say, a time-dependent magnetic field. What counts are the complete dynamical orbits in S. They represent the physical reality. To generate all the orbits that pass through a given point in $\mathbf{S}$, one can always adopt the convention that $\tau=1$ in the formulation of the initial-value problem. In it, one specifies $6 N-14$ rotationally invariant components of $\boldsymbol{\sigma}_{a}(1)$ and $\boldsymbol{\pi}_{a}(1)$; each choice of them will generate a different curve in S. Taken together, all these initial conditions generate all of the dynamical curves that pass through the given point. Nothing can change them. This is quite unlike what happens to dynamical evolution subject to time-dependent external fields.

Note also that in Newtonian terms the $\boldsymbol{\pi}_{a}(1)$ are the dimensionless ratios of the shape-changing momenta divided by the momentum $D_{0}$ in overall initial expansion.

The theory defined by the Hamiltonian (15) satisfies the weak Mach-Poincaré principle because, given $\boldsymbol{\sigma}_{a}(1)$ and $\boldsymbol{\pi}_{a}(1)$, the equations of motion (16) generate a unique curve in $\mathrm{S}$. We can construct the Newtonian trajectory in the extended phase space $\left(\mathbf{r}_{a}, \mathbf{p}^{a}\right)$ by inverting (9) to obtain the scaled data $D(\tau)$ and $R(\tau)$ in Eq. (14). We must also specify $D_{0}$, but this, our only dimensionful quantity (it gives length dimensions to everything else), amounts to the conventional choice of a unit. ${ }^{5}$

If $E \neq 0$, we need an extra initial datum: the dimensionless $\epsilon=E D_{0}^{2}$, which appears in the Hamiltonian:

$$
\mathcal{H}=\log \left[V_{\mathrm{s}}+\sqrt{V_{\mathrm{s}}^{2}+\frac{1}{2} \epsilon\left(K_{\mathrm{s}}+\tau^{2}\right)}\right] .
$$

\footnotetext{
${ }^{5}$ We have already restricted the choice of units in the Newtonian representation by setting the Newton constant $G=1$ and using 'geometrical' masses.
} 
The Mach-Poincaré principle fails due to the necessity to specify $\epsilon \tau^{2}$, but the theory is still scale invariant and fully dimensionless with a monotonic independent variable. Our solution to the problem of time still holds.

\section{Geometrodynamic Shape Dynamics}

From the point of view of the problem of time, the vitally important feature of geometrodynamic SD is that it completely eliminates many-fingered evolution and gives Einsteinian gravitational dynamics an architectonic structure essentially identical to $N$-body dynamics. As shown in [7], it does this by trading all but one linear combination of the quadratic constraints at each space point in the integral (2) for a volume-preserving conformal constraint

$$
g_{a b} p^{a b}-\sqrt{g} Y=0,
$$

which together with the diffeomorphism constraints (3) reduces the configuration space to $\mathbf{S} \times \mathbb{R}^{+} ; \mathbf{S}$ is conformal superspace $\mathbf{C}$, the geometrodynamic shape space, and $\mathbb{R}^{+}$represents the volume $V=\int d^{3} x \sqrt{g}$, which is invariant under the transformations generated by (18) and, at this stage, a dynamical degree of freedom; $Y=\int d^{3} x g_{a b} p^{a b} / V$ is proportional to 'York time' and is monotonic along spacetime solutions that can be foliated by spacelike hypersurfaces of constant-mean-(extrinsic) curvature (CMC) [13]. ${ }^{6}$ The volume and $Y$ are canonical conjugates: $\{V, Y\}=\frac{3}{2}$.

Here too we find it convenient not to solve the linear (diffeo) constraint, and we work in pre-shape space plus volume: $P S \times \mathbb{R}^{+}$, where PS $=$Riem $/$Conf .

The symmetry trading procedure allows one to trade the constraints (2) for (18) with the exception of one single constraint. This eliminates the many-fingered evolution, the residual constraint becoming a reparametrization constraint for dynamics on $\mathrm{PS} \times \mathbb{R}^{+}$. Exactly as before, we now describe the dynamics on PS, using $Y$, like $\tau$, as independent variable and $V$ as physical Hamiltonian. Solved for $V$, the residual constraint gives

$$
\mathcal{H}\left[g_{a b}, p^{a b}, Y\right]=\int d^{3} x \sqrt{g} \phi^{6}\left[g_{a b}, p^{a b}, Y ; x\right),
$$

where $\phi$ solves the Lichnerowicz-York (LY) equation (which always has a unique solution [20])

$$
\frac{g_{a c} g_{b d} p_{\mathrm{T}}^{a b} p_{\mathrm{T}}^{c d}}{\phi^{12} g}-\phi^{-4}\left(R-8 \phi^{-1} \nabla^{2} \phi\right)-\frac{1}{6} Y^{2}=0,
$$

$p_{\mathrm{T}}^{a b}=p^{a b}-\frac{1}{3} g^{a b} p^{c d} g_{c d}$ is the traceless part of $p^{a b}$.

The Hamiltonian (19) is obviously diffeo-invariant and also fully conformally invariant if $Y$ is treated as the independent variable,

$$
\mathcal{H}\left[\omega^{4} g_{a b}, \omega^{-4} p^{a b}, Y\right]=\mathcal{H}\left[g_{a b}, p^{a b}, Y\right],
$$

\footnotetext{
${ }^{6}$ In vacuum GR, the analogue of the passage from the coordinatized to the objective description of Newtonian dynamics that we described in Sec. 4 amounts to identification of the conformal threegeometries on successive CMC slices of a spatially closed Einsteinian spacetime and projection of them to conformal superspace, where they form a unique curve that we regard as the entire objective content of theory.
} 
because (20) shows that $\phi\left[g_{a b}, p^{a b}, Y ; x\right)$ transforms as

$$
\phi\left[\omega^{4} g_{a b}, \omega^{-4} p^{a b}, Y ; x\right)=\omega^{-1}(x) \phi\left[g_{a b}, p^{a b}, Y ; x\right),
$$

while the $\sqrt{g}$ factor in (19) transforms as $\sqrt{g} \rightarrow \omega^{6} \sqrt{g}$, cancelling $\omega$. Further, as noted in [14], the LY equation (20), like the $N$-body equations, exhibits a form of dynamical similarity, which we here express in the more convenient equivalent form

$$
\phi\left[g_{a b}, \alpha^{4} p^{a b}, \alpha^{-2} Y ; x\right)=\alpha \phi\left[g_{a b}, p^{a b}, Y ; x\right),
$$

where $\alpha$ is a spatial constant. This means that the SD Hamiltonian scales covariantly with $\alpha^{6}$ :

$$
\mathcal{H}\left[g_{a b}, \alpha^{4} p^{a b}, \alpha^{-2} Y\right]=\alpha^{6} \mathcal{H}\left[g_{a b}, p^{a b}, Y\right] .
$$

Note that the ADM Hamiltonian (2) has an indefinite kinetic energy due to the $-\frac{1}{2} p^{2}$ term. Our description completely removes this long-standing conformal-factor problem. The volume-preserving conformal constraint (18) makes most of the positiondependent $-\frac{1}{2} p^{2}$ term pure gauge. The remaining part, the spatial average of $p$, is used as the independent variable and therefore removed from the dynamics. We are left with an SD Hamiltonian with non-negative kinetic energy.

For the dimensional analysis, we use Dicke's convention [21], in which the 3metric has $\left[g_{i j}\right]=\ell^{2},\left[g^{i j}\right]=\ell^{-2}$, while the coordinates (and, accordingly, space derivatives) are dimensionless labels for points. The momenta are dimensionless $\left[p^{i j}\right]=1$, the York time has $[Y]=\ell^{-1}$.

We want to express the dynamics in terms of dimensionless and conformally invariant variables on the phase space of pre-shape space PS. By analogy with the particle model, we choose the unimodular metric $\tilde{g}_{a b}=g_{a b} / g^{\frac{1}{3}}$ (analogous to $\boldsymbol{\sigma}_{a}$ ) and dimensionless shape momenta $\tilde{p}_{\mathrm{T}}^{a b}=Y_{0}^{2} g^{\frac{1}{3}} p_{\mathrm{T}}^{a b}$, which have exactly the same structure as $\boldsymbol{p}^{a}$ : they are obtained by multiplying the traceless part of $p^{a b}$ by $g^{\frac{1}{3}}$ (the analogue of $R$ ) to make it conformally invariant, and then multiplying by the appropriate power of $Y_{0}$, an initial value of $Y$ (analogous to $D_{0}$ ), to make it dimensionless. In analogy with Eq. (11), the shape variables $\left(\tilde{g}_{a b}, \tilde{p}^{a b}\right)$ Poisson commute with every functional of $Y$ and $V$,

$$
\left\{F[Y, V], \tilde{g}_{a b}\right\}=\left\{F[Y, V], \tilde{p}^{a b}\right\}=0,
$$

and satisfy constraints analogous to (10):

$$
\operatorname{det} \tilde{g}_{a b}=1, \quad \tilde{g}_{a b} \tilde{p}^{a b}=0 .
$$

The symplectic structure on S perfectly matches that of the particle model (12),

$$
\begin{aligned}
& \left\{\tilde{g}_{a b}(x), \tilde{p}_{\mathrm{T}}^{c d}(y)\right\}=Y_{0}^{2}\left(\frac{1}{2} \delta_{(a}^{c} \delta_{b)}^{d}-\frac{1}{3} \tilde{g}_{a b} \tilde{g}^{c d}\right) \delta(x, y), \\
& \left\{\tilde{p}_{\mathrm{T}}^{a b}(x), \tilde{p}_{\mathrm{T}}^{c d}(y)\right\}=\frac{1}{3} Y_{0}^{2}\left(\tilde{g}^{c d} \tilde{p}_{\mathrm{T}}^{a b}-\tilde{g}^{a b} \tilde{p}_{\mathrm{T}}^{c d}\right) \delta(x, y), \\
& \left\{\tilde{g}_{a b}(x), \tilde{g}_{c d}(y)\right\}=0 .
\end{aligned}
$$

The SD Hamiltonian has the dimensions of a volume, $[\mathcal{H}]=\ell^{3}$, the Poisson brackets an inverse area $[\{.,\}]=.\ell^{-2}$. 
We now exploit the two invariances (21) and (23) to write a dimensionless Hamiltonian $\tilde{\mathcal{H}}$ on PS that generates evolution in the dimensionless independent variable $\tau=Y / Y_{0}$ :

$$
\tilde{\mathcal{H}}\left[\tilde{g}_{a b}, \tilde{p}^{a b}, \tau\right]=\int d^{3} x \tilde{\phi}^{6}\left[\tilde{g}_{a b}, \tilde{p}_{\mathrm{T}}^{a b}, \tau ; x\right),
$$

where $\tilde{\phi}$ is a scalar density of weight $\frac{1}{6}$ (it contains a factor of $g^{\frac{1}{12}}$ ) and solves the dimensionless equation

$$
\frac{\tilde{g}_{a c} \tilde{g}_{b d} \tilde{p}_{\mathrm{T}}^{a b} \tilde{p}_{\mathrm{T}}^{c d}}{\tilde{\phi}^{12}}-\tilde{\phi}^{-4}\left(\tilde{R}-8 \tilde{\phi}^{-1} \tilde{\nabla}^{2} \tilde{\phi}\right)-\frac{1}{6} \tau^{2}=0,
$$

where $\tilde{R}=R\left(\tilde{g}_{a b}\right)=g^{\frac{1}{3}} R\left(g_{a b}\right)$, and $\tilde{\nabla}^{2}=\tilde{g}^{a b} \nabla_{a} \nabla_{b}$.

A functional $F\left[\tilde{g}_{a b}, \tilde{p}^{a b}\right]$ on PS then evolves as

$$
\frac{d}{d \tau} F\left[\tilde{g}_{a b}, \tilde{p}^{a b}\right]=\frac{2}{3} Y_{0}^{-2}\left\{\tilde{\mathcal{H}}\left[\tilde{g}_{a b}, \tilde{p}^{a b}, \tau\right], F\left[\tilde{g}_{a b}, \tilde{p}^{a b}\right]\right\}
$$

and, as in the particle model, the $Y_{0}^{-2}$ term on the rhs compensates for the corresponding factor in (27).

As York showed [22, 20, 13], the diffeomorphism constraint decouples from the conformally invariant degrees of freedom and can be solved independently. This happens because the equations of motion in PS are diffeo-invariant and, in turn, the diffeomorphism constraint is conformally covariant. This parallels the particle model, for which the dynamics in $\mathbf{S}$ is rotationally invariant and the angular-momentum constraint is scale invariant.

In the initial-value problem on $\mathbf{S}$, one specifies a unimodular 3-geometry $\left(\tilde{g}_{a b}\right.$ modulo diffeos) and unconstrained transverse-traceless momenta $\tilde{p}_{\mathrm{TT}}^{a b}$. These are 4 degrees of freedom per point. The Hamiltonian $\tilde{\mathcal{H}}$ then generates a unique curve in $\mathbf{S}$, parametrized by $\tau$. This system satisfies the weak Mach-Poincaré principle. The ADM description is obtained by introducing the determinant $g=$ $Y_{0}^{-6} \tilde{\phi}^{12}\left[\tilde{g}_{a b}, \tilde{p}_{\mathrm{T}}^{a b}, \tau ; x\right)$. Like $D_{0}$ in the particle model, the value given to $Y_{0}$ is conventional. All dimensions in the ADM representation arise from it.

\section{Extensions}

The bosonic matter of the standard model can be included in this picture by requiring the matter fields to transform with conformal weight zero [23]. The two key properties (22) and (23) still hold, so the result goes through unchanged. The only exception to this is a cosmological constant $\Lambda$. It resembles the $E \neq 0$ case in the particle model. The LY equation gains a constant term in addition to $Y^{2}$, and (23) no longer holds (this happens also for a Higgs potential): one must rescale $\Lambda$ and specify the additional dimensionless quantity $\lambda=\Lambda / Y_{0}^{2}$, so the Mach-Poincaré principle fails.

This failure in no way affects our solution to the problem of time. It is rather to be seen as a potential criterion for theory selection. Einstein was always struck by the fact that GR is the simplest non-trivial theory of dynamical Riemannian geometry. Faith in simplicity would lead one to seek to explain the apparent existence of a cosmological constant $\Lambda$ through some process by which it emerges from 
a fundamental theory that does not contain it. In this connection, our assumptions of positive energy in the $N$-body problem and positive $\Lambda$, under which $\tau$ is certainly monotonic, are mild and supported observationally - a fundamental $\Lambda$, if it does exist, is positive.

\section{Conclusions}

We believe that, taken together, the results of this paper solve the long-standing classical problem of time and are a promising basis for the quantum treatment. In Einsteinian gravity, the solution consists of two stages: disentanglement of the evolution from gauge followed by deparametrization. In the $N$-body problem, only deparametrization is needed. Let us recapitulate the key aspects.

1. Geometrodynamic SD as established in [7] cleanly disentangles the true dynamical evolution in gravity from the gauge invariance associated with the coordinatized spacetime description, reducing the problem of time to reparametrization invariance. The disentanglement is simultaneously the key step to the representation of Einsteinian gravity in dimensionless form, the sine qua non for the subsequent introduction of $\tau$ and the full solution to the problem of time. That simply cannot be done without the passage from Wheeler's metric-based superspace, with its three degrees of freedom per space point, to metric-free conformal superspace, which, as York [13] noted, is dimensionless and has two degrees of freedom per space point. ${ }^{7}$

2. After this step, closed-space geometrodynamic SD, just like the Newtonian $N$-body problem, admits an objective description in completely dimensionless and scale-invariant terms. The evolution takes place wrt an unambiguously defined monotonic independent variable. ${ }^{8}$ The observational equivalence of what we have called the coordinatized and objective descriptions ensures that this solves the problem of time.

We also want to recall the observation made after (24), which is that the ADM kinetic energy has a negative part. It has been a problematic issue for quantumgravity research for decades, especially approaches based on path integrals, which as a result are unbounded below. This conformal mode problem has been considered in many approaches, see e.g. Hořava's [24] modified kinetic term. In our treatment,

\footnotetext{
${ }^{7}$ We should place on record that our paper, like all previous work on SD, draws heavily on York's work, in which our collaborator Ó Murchadha played an important role. In fact, York came rather close to our solution to the problem of time. However, his analysis was based on full conformal transformations, not the volume-preserving transformations generated by (18). He also did not aim for a dimensionless 'time', settling instead for the dimensionful York time. This is probably because he did not have the advantage of knowing the dynamical similarity (23) of the LY equation, which was first recognized by Ó Murchadha [14] and led to our idea of introducing a dimensionless independent variable.

${ }^{8}$ This statement is subject to the minor caveat noted in footnote 4: any monotonic function $f$ of $\tau$ will serve equally well. This explains why our independent variable is not related to the readings of physical clocks. The change from $\tau$ to any monotonic $f(\tau)$ multiplies the Hamiltonian by a corresponding lapse. The evolution curves in Shape Space are unchanged. If any $f(\tau)$ is in any way distinguished it is $T=\log (\tau)$, which allows one to set initial data at $T=0$ but perhaps more significantly makes the Hamiltonian $T$-independent in interesting asymptotic regimes. This might have technical advantages. But fundamentally no specific choice of $f(\tau)$ has any ontological priority. We have to make some choice and with it a corresponding Hamiltonian to express the law that generates the successive shapes in S. The shapes alone are ontological.
} 
the troublesome contribution divides into a local part, which is pure gauge, and a spatial average which, made dimensionless, plays the role of the independent variable in our description and, as we have already noted, ceases to be dynamic. Thus, the kinetic-energy part of the physical Hamiltonian is shown to be positive definite. This appears to be a major bonus that comes with our solution to the problem of time.

To end, we should like to add some further arguments for the dimensionless representation of both Newtonian and Einsteinian gravity.

Physicists are widely agreed that the results of laboratory experiments or theoretical calculations must, if they are to be objective, be expressed in dimensionless form. This requirement, whose extension to a dynamically closed universe can hardly be questioned, is the sole principle that underlies our identification of the independent variable $\tau$ once gauge has been disentangled from dynamics.

Moreover, the elimination of redundancy and convention, especially external scale, reveals more than one might expect. One sees this already in Newtonian dynamics. Far from being the single theory it appears to be in the familiar representation, it is actually three: one with energy $E=0$ and two more, with $E<0$ (which we have not considered) and $E>0$. These theories are governed by very different Hamiltonians (compare (15) and (17)). Furthermore, a dynamically closed Newtonian universe cannot have different energies; it can just have one of those three different governing laws. There no such thing as a conserved energy of a universe with different possible values determined by initial conditions. Finally, time as deeply rooted in intuition as the 19th-century aether - is simply not there. The only 'time' we can find is $D / D_{0}$.

If passage to the dimensionless description puts Newtonian 'spacetime' in such a different light, we can surely expect the same to happen with Einsteinian spacetime. The way GR is normally taught is that extremalization of the Einstein-Hilbert action selects among all pseudo-Riemannian manifolds, each with a complete fourdimensional metric $g_{\mu \nu}$, those that are candidates for physical realization. However, we start with a vastly more restricted ontology: three-dimensional conformal geometries. We then postulate that a point and a tangent vector in conformal superspace, C, must uniquely determine an evolution curve in C.

This bare input is sufficient to 'create' an entire Einsteinian spacetime endowed with local proper distance, local proper time and local inertial frames of reference. They are artefacts of the fundamental law that generates the curve of shapes in C and disappear when we return to the objective description as outlined in footnote 6 . We only think they are there because of the way the fundamental law makes one shape follow another, picking out a very special curve in C in doing so [25].

If progress requires us to give up relativity of simultaneity, viewing the status it has acquired as historically contingent, we think the price is worth paying. Let us remind the reader of Dirac's words in 1958 [26], who was so struck by the simplicity of the Hamiltonian treatment obtained by giving up the spacetime description that he commented: "I am inclined to believe from this that four-dimensional symmetry is not a fundamental property of the physical world." We would slightly modify that and say the appearance of four-dimensional symmetry is a by-product of the way one shape follows another.

Acknowledgements. TK was supported in part through NSERC. JB was 
supported by a grant from the Foundational Questions Institute (FQXi) Fund, a donor advised fund of the Silicon Valley Community Foundation on the basis of proposal FQXi Time and Foundations 2010 to the Foundational Questions Institute. FM was supported by a grant from the John Templeton Foundation. The opinions expressed in this publication are those of the authors and do not necessarily reflect the views of the John Templeton Foundation. Research at Perimeter Institute is supported by the Government of Canada through Industry Canada and by the Province of Ontario through the Ministry of Economic Development and Innovation. We thank Boris Barbour for helpful comments on the text.

\section{References}

[1] E. Anderson, "The problem of time in quantum gravity," arXiv:1009.2157 [gr-qc].

[2] P. A. M. Dirac, "Fixation of coordinates in the Hamiltonian theory of gravitation," Phys. Rev. 114 (1959) 924-930.

[3] M. Bojowald, P. A. Höhn, and A. Tsobanjan, "An effective approach to the problem of time," Class.Quant.Grav. 28 (2011) 035006, arXiv:1009.5953 [gr-qc].

[4] J. Barbour and B. Bertotti, "Mach's principle and the structure of dynamical theories," Proc. R. Soc. A 382 no. 1783, (1982) 295-306.

[5] J. Barbour and N. O’Murchadha, "Classical and quantum gravity on conformal superspace," arXiv:gr-qc/9911071.

[6] E. Anderson, J. Barbour, B. Z. Foster, B. Kelleher, and N. O'Murchadha, "The physical gravitational degrees of freedom," Class. Quant. Grav. 22 (2005) 1795-1802, arXiv:gr-qc/0407104.

[7] H. Gomes, S. Gryb, and T. Koslowski, "Einstein gravity as a 3D conformally invariant theory," Class. Quant. Grav. 28 (2011) 045005, arXiv:1010.2481 [gr-qc] .

[8] H. Poincaré, Science et Hypothèse. Paris, 1902.

[9] J. Barbour, "The definition of Mach's principle," Found. Phys. 40 (2010) 1263-1284, arXiv:1007.3368 [gr-qc].

[10] J. A. Wheeler, "Superspace and the nature of quantum geometrodynamics," Battelle Rencontres (1967) 242-307.

[11] D. Giulini, "The superspace of geometrodynamics," Gen. Rel. Grav. 41 (2009) 785-815, arXiv:0902.3923 [gr-qc].

[12] James W. York, Jr., "Gravitational degrees of freedom and the initial-value problem," Phys. Rev. Lett. 26 (1971) 1656-1658. 
[13] J. James W. York, Jr., "Role of conformal three geometry in the dynamics of gravitation," Phys. Rev. Lett. 28 (1972) 1082-1085.

[14] J. Barbour and N. O'Murchadha, "Conformal Superspace: the configuration space of general relativity," arXiv:1009.3559 [gr-qc].

[15] T. Koslowski, "Observable equivalence between general relativity and shape dynamics," arXiv:1203.6688 [gr-qc].

[16] J. Barbour, "Shape dynamics. An introduction," in Quantum Field Theory and Gravity. Proc. Conference at Regensburg 2010, F. Finster, ed. Birkhäuser, 2012. arXiv: 1105.0183.

[17] J. Barbour, M. Lostaglio, and F. Mercati, "Scale anomaly as the origin of time," arXiv:1301.6173 [gr-qc].

[18] D. G. Saari, Collisions, Rings, and other Newtonian N-body Problems. American Mathematical Society, Providence, RI, 2005.

[19] L. D. Landau and E. M. Lifshitz, Course of theoretical physics, vol. 1: Mechanics, ch. II, sec. 10. Pergamon Press, Oxford, 1976.

[20] N. O’Murchadha and J. James W. York, Jr., "Existence and uniqueness of solutions of the Hamiltonian constraint of general relativity on compact manifolds," J. Math. Phys. 14 (1973) 1551-1557.

[21] R. Dicke, Relativity, Groups, and Topology. Gordon and Breach, NY, 1964.

[22] J. James W. York, Jr., "Conformally invariant orthogonal decomposition of symmetric tensors on Riemannian manifolds and the initial value problem of general relativity," J. Math. Phys. 14 (1973) 456-464.

[23] H. Gomes and T. Koslowski, "Coupling shape dynamics to matter gives spacetime," Gen. Rel. Grav. 44 (10, 2012) 1539-1553, arXiv:1110.3837 [gr-qc].

[24] P. Hořava, "Quantum gravity at a Lifshitz point," Phys. Rev. D79 (2009) 084008, arXiv:0901.3775 [hep-th].

[25] J. Barbour, "Reductionist doubts." Third-prize winning essay for the fqxi essay contest 'questioning the foundations', 2012. http://fqxi.org/community/essay/winners/2012.1\#barbour.

[26] P. A. M. Dirac, "Generalized Hamiltonian dynamics," Proc. R. Soc. A 246 (1958) 326-332. 